\title{
Construction and Analysis of Quaternion MIMO-OFDM Communications Systems
}

\author{
L.G.P. Meloni, J.L. Hinostroza N., and O. Tormena Jr.
}

\begin{abstract}
Wireless communications systems with progressively higher spectral efficiency have been investigated in past decades. A promising area of research is the use of hypercomplex algebras, notably, the use of the quaternion algebra. This paper considers the construction of multiple-input-multiple-output (MIMO) orthogonal frequency division multiplexing (OFDM) using the algebra of quaternions. Several construction techniques for quaternion orthogonal code designs have been proposed in recent years, which offer the possibility to explore diversities in various domains, such as space, time, frequency, and polarization, in addition to combinations thereof. This paper presents a formulation for quaternion MIMO-OFDM in matrix form as an extension of the classical formulation that uses complex variables. Quaternions allow elegant representation of pairs of radiant elements in physical antennas configured for crosspolarized propagation. Several simulations validate the proposed method in diverse scenarios for wireless communications, in which combined diversities have been exploited.
\end{abstract}

Index Terms-Quaternion MIMO-OFDM, quaternion Fourier transform, combined diversities, frequency-selective fading channels, cross-polarized antennas.

\section{INTRODUCTION}

The search for wireless communications systems with better spectral efficiency or low-complexity has continued over recent decades. One promising field for this aim is the use of hypercomplex algebras, among which we highlight the quaternions. Quaternions have attracted the attention of researchers in many fields of engineering in past decades. In present times, the most widespread practical applications of quaternions have been in $3 \mathrm{D}$ animation to solve the problem called gimbal lock instability. This instability occurs when the orientation of a sensor cannot be determined uniquely by a mathematical representation using Euler angles, in which case, the 3D system looses one degree of freedom [1]. In inertial navigation systems, the application of quaternions using sensors (gyroscope and accelerometers) in aerospace vehicles has facilitated the study of these dynamic systems and error propagation equations [2].

Quaternion Laplace and Fourier transforms are more recent, dating from beginning of 1990s [3]. However, spectral representations with hypercomplex commutative algebra were developed prior to this work; they have appeared in 2D imaging

L.G.P. Meloni and J.L.Hinostroza N. are with School of Electrical and Computer Engineering, University of Campinas, Campinas, SP, Brazil.

E-mail: meloni@decom.fee.unicamp.br, hinos.jl@ decom.fee.unicamp.br

O. Tormena Jr. is with Academic Department of Electronics, Federal Technology University of Paraná, Campo Mourão, PR, Brazil.

E-mail: tormena@utfpr.edu.br

The Associate Editor coordinating the review of this manuscript and approving it for publication was Prof. Renato Machado.

Digital Object Identifier: 10.14209/jcis.2017.9 studies in nuclear magnetic resonance [4]. In 1998, Sangwine and Ell concluded that an arbitrary root of -1 in 3D space could be used as the imaginary unit in the quaternion Fourier transform, which also allows one to apply this transform to 1D signals [5]. The representation of a quaternion in its polar form allows the formulation of instant frequency and amplitude modulation of ortho-complex signals based on the quaternion Hilbert transform [6]. Another area of interest is the application of quaternions for colour images, which are now treated as vector fields. Ell and Sangwine [7] have shown that the quaternion Fourier transform is appropriate for describing the spectral content of images. They have also shown applications of quaternions for linear filtering of colour images, which have no equivalent forms with iterated filters when using separate colours.

The applications of quaternions in wireless communications have also arisen at the frontier of knowledge. Quaternion algebra is well suited for the description of MIMO channels, for which antenna elements are configured for the crosspolarization operation [8]. Quaternions for diversity schemes for increased spectral efficiency has also been studied in the design of space-time-polarization codes [9]. In a recent study, one of the authors has introduced the use of quaternions and hypercomplex commutative algebras to OFDM modulation schemes in [10]. It was shown that the one-dimensional quaternion Fourier transform is applicable to OFDM schemes.

The concept of MIMO-OFDM was first described by Rayleigh and Jones before the turn of the century [11]. The first widespread application of the technology was in the IEEE 802.11n standard [12]. The application of concepts from MIMO-OFDM are also responsible for the diversity gains observed in current technologies, like IEEE 802.11ac, IEEE 802.16 (WiMAX) and LTE (Long Term Evolution) mobile, as well as expected gains for future technologies like IEEE 802.11ax and 5G mobile [13].

In this paper, we extended MIMO diversity coding to quaternion OFDM. MIMO has been introduced to complex OFDM as a direct extension of Alamouti space-time coding to this modulation technique in beginning of 2000s [14], [15]. The importance of MIMO transmission with crosspolarization antenna elements can be observed in recent field tests conducted by Japanese public radio broadcasting (NHK) [16]. Tests for terrestrial digital television signals in Super HiVision (8K) format have successfully reported transmission over long distances, showing that it is possible to send huge amounts of data in the 6-MHz UHF channel. These results also motivate the present work, in which we show that MIMOOFDM modulation schemes can be incorporated in systems for next-generation digital television, in addition to having 
potential applications in high-capacity link back-hauling and other wireless applications.

In typical MIMO communication systems, as described in [17], antenna spacings of at least half of the link wavelength at the subscriber unit and ten wavelengths at the base station are required to achieve significant multiplexing and/or diversity gains. Thus, the possible use of co-located orthogonally polarized antennas is an effective alternative for space and cost reduction. Another possibility in these communications schemes (which do not use cross-polarized antennas) is to employ a pair of spatially separated antennas at both the transmit and receive sides. MIMO-OFDM systems, presented in the paper, allow to explore diversity modalities in space, time, frequency, and/or polarization, offering very flexible communications designs. Several simulation scenarios are presented in paper, including comparision to complex cases, show the potential for improving the above-mentioned gains and further allowing use of highly efficient orthogonal code designs.

This article is organized as follows. In Section 2, classical MIMO-OFDM is reviewed for space-time and space-frequency diversities, followed by a review of OFDM systems using quaternion mathematics. Section 3 presents a formulation of quaternion MIMO-OFDM, several quaternion orthogonal designs, and the maximum likelihood decoders. In Section 5 , we present several simulation studies in different wireless communications scenarios showing the advantages of using quaternions for diversities in variate domains. Finally, Section 6 concludes the paper.

\section{ClassiCAL MIMO-OFDM SystemS}

OFDM is a technique for digital modulation that is widely used in wireless communications. It is typically implemented using discrete Fourier transforms (DFTs) yielding high data rates in frequency-selective fading channels. OFDM is applied to many modern communications systems such as wireless mobile communications, broadcasting of digital radio and television signals, and wireless LAN. The discrete Fourier transform can also be viewed as a modulation technique with several transmit subcarriers, which are equally spaced in frequency; these subcarriers are defined by the base functions of the transform. For transmission, not all subcarriers are used for data modulation: some are reserved as guard frequencies to provide robustness against interference from adjacent channels $\left(N_{g}\right)$, and some are reserved for synchronization of pilot subcarriers $\left(N_{p}\right)$. Pilot carriers are also used for channel equalization. Message bit-streams are grouped for modulating subcarriers of all other Fourier transform base functions (consisting of $N_{l}$ payload subcarriers).

In the transmitter, serial-to-parallel data conversion allows mapping of the message bit-stream according to the constellation in the modulation, so as to form a discrete-Fourier transform vector of size $N=N_{l}+N_{g}+N_{p}$. Normally, $N$ is chosen to be a power of 2 to take the advantage of fast Fourier transform algorithms. Thus, OFDM can be viewed as a block or symbol vector transmission system. For each OFDM symbol, $N$ carriers are prepared for computing the inverse
Fourier transform. Therefore, this vector is time dependent, changing at $m T_{s}$ for each OFDM symbol period $T_{s}$, and containing $N$ subcarriers, that is

$$
\boldsymbol{X}_{m}=\left[\begin{array}{llll}
X_{m}[0] & X_{m}[1] & \ldots & \left.X_{m}[N-1]\right]^{\mathcal{T}},
\end{array}\right.
$$

where $\mathcal{T}$ represents the vector transpose. This vector is then submitted to the inverse Fourier transform. In the time domain, the last $N_{c}$ block samples are repeated at its beginning, creating a cyclic extension which is used as a time guard interval. Typically, $N_{c}$ is chosen to be $1 / 4,1 / 8,1 / 16$, or $1 / 32$ of the size $N$ of the FFT. Therefore, in the time domain, the OFDM symbol has a length of $N_{s}=N+N_{c}$, which is cyclic extended into a vector

$$
\boldsymbol{x}_{m}^{c}=\left[x_{m}\left[N-N_{c}\right] \ldots x_{m}[N-1] x_{m}[0] \ldots x_{m}[N-1]\right]^{\mathcal{T}} \text {. }
$$

It is important to note that it is possible to use different modulation techniques for different groups of subcarriers. Note that during the period $T_{s}=N_{s} T$, the vector $\boldsymbol{x}_{m}^{c}$ is serially transmitted by the sequence $x[n]$, where $T$ is the sampling period.

In typical OFDM modulation, the signal sequence $x[n]$ is transmitted through a frequency-selective fading channel of order $L$, such that the causal response of the channel link is $h_{m}[n]=0$, for $n<0$ and for $n>L$. To avoid inter-symbol interference, the time guard interval is $N_{c} \geq L$. Typically, we assume that the channel response is invariant during the OFDM symbol period, in which case, the receive signal is simply the convolution of $h_{m}[n]$ with the sequence $x[n]$, i.e., $y[n]=x[n] * h_{m}[n]$; however, $h_{m}[n]$ varies for each symbol $m$. At the receiver side, the sequence $y[n]$ is segmented symbolto-symbol, resulting in the sequence $y_{m}^{c}[n]$.

After removing the cyclic samples of the time guard interval, one obtains a received time vector of size $N$

$$
\boldsymbol{y}_{m}=\left[\begin{array}{llll}
y_{m}^{c}\left[N_{c}\right] & y_{m}^{c}\left[N_{c}+1\right] & \ldots & y_{m}^{c}\left[N_{c}+N-1\right]
\end{array}\right]^{\mathcal{T}} .
$$

Convolution in the time domain corresponds to the product in the DFT domain, that is, for the cyclic-prefix-removed sequences,

$$
Y_{m}[k]=X_{m}[k] H_{m}[k]+Z_{m}[k], \quad k=0,1, \ldots, N-1,
$$

where $H_{m}[k]$ is the DFT of $h_{m}[n]$, and $Z_{m}[k]$ is the DFT of the channel noise at OFDM symbol $m$. This expression can be expressed in matrix form as

$$
\boldsymbol{Y}_{m}=\mathbf{X}_{m} \boldsymbol{H}_{m}+\boldsymbol{Z}_{\boldsymbol{m}},
$$

where $\mathbf{X}_{m}$ is a diagonal matrix whose elements are the DFT sequence $X_{m}[k], \boldsymbol{H}_{\boldsymbol{m}}$ and $\boldsymbol{Z}_{\boldsymbol{m}}$ are respectively the frequency response of the channel and the DFT of the channel noise, both refered to the OFDM symbol $m$. This equation means that the OFDM with a cyclic prefix transforms a frequency-selective fading channel into $N$ perfectly flat fading sub-channels.

\section{A. Transmit Diversity in Time Domain}

MIMO technique has been introduced to classical OFDM. The direct extension of Alamouti space-time coding to this modulation [14] requires two OFDM symbols, as shown in 
Fig. 1. This code application achieves diversity gains over frequency-selective fading channels. Note that this scheme will use one OFDM engine for each transmit antenna. Therefore, after the serial-to-parallel conversion block, two data blocks are created

$$
\begin{gathered}
\mathbf{X}_{m}=\operatorname{diag}\left\{X_{m}[0], X_{m}[1], \ldots, X_{m}[N-1]\right\}, \\
\mathbf{X}_{m+1}=\operatorname{diag}\left\{X_{m+1}[0], X_{m+1}[1], \ldots, X_{m+1}[N-1]\right\} .
\end{gathered}
$$

At the upper transmitter in the first OFDM symbol time slot, $\mathbf{X}_{m}$ is transmitted, followed by $-\mathbf{X}_{m+1}^{*}$ in the second time slot. At the second transmitter, $\mathbf{X}_{m+1}$ is transmitted first, followed by $\mathbf{X}_{m}^{*}$.

The equivalent space-time block code transmit matrix is

$$
\mathbf{C}=\left[\begin{array}{cc}
\mathbf{X}_{m} & \mathbf{X}_{m+1} \\
-\mathbf{X}_{m+1}^{*} & \mathbf{X}_{m}^{*}
\end{array}\right],
$$

whose elements are the OFDM symbol matrices and its conjugates. The first row of the matrix $\mathbf{C}$ corresponds to time slot $m$ and the second row corresponds to time slot $m+1$; the first column of matrix $\mathbf{C}$ corresponds to the signal transmitted from the $1^{\text {st }}$ antenna and the second column from the $2^{\text {nd }}$ antenna. Because $\boldsymbol{H}_{m}^{(1,1)}$ and $\boldsymbol{H}_{m}^{(2,1)}$ are the respective DFTs of the channel unit responses $h_{m}^{(1,1)}[n]$ and $h_{m}^{(2,1)}[n]$, where $h_{m}^{(r, s)}[n]$ represents the channel fading gain of the link from transmit antenna $r$ to receive antenna $s$, and because these responses are constant during two consecutive OFDM symbol periods, the correspondent received vectors is given by $\boldsymbol{Y}=\mathbf{C H}+\boldsymbol{Z}$, or, explicitly,

$$
\left[\begin{array}{c}
\boldsymbol{Y}_{m} \\
\boldsymbol{Y}_{m+1}
\end{array}\right]=\left[\begin{array}{cc}
\mathbf{X}_{m} & \mathbf{X}_{m+1} \\
-\mathbf{X}_{m+1}^{*} & \mathbf{X}_{m}^{*}
\end{array}\right]\left[\begin{array}{c}
\boldsymbol{H}_{m}^{(1,1)} \\
\boldsymbol{H}_{m}^{(2,1)}
\end{array}\right]+\left[\begin{array}{c}
\boldsymbol{Z}_{m} \\
\boldsymbol{Z}_{m+1}
\end{array}\right]
$$

where $Z_{m}$ and $Z_{m+1}$ are noise components. By rearranging the above equation, one can write

$$
\left[\begin{array}{c}
\boldsymbol{Y}_{m} \\
\boldsymbol{Y}_{m+1}^{*}
\end{array}\right]=\left[\begin{array}{cc}
\mathbf{H}_{m}^{(1,1)} & \mathbf{H}_{m}^{(2,1)} \\
\mathbf{H}_{m}^{(2,1) *} & -\mathbf{H}_{m}^{(1,1) *}
\end{array}\right]\left[\begin{array}{c}
\boldsymbol{X}_{m} \\
\boldsymbol{X}_{m+1}
\end{array}\right]+\left[\begin{array}{c}
\boldsymbol{Z}_{m} \\
\boldsymbol{Z}_{m+1}^{*}
\end{array}\right],
$$

where $\mathbf{H}_{m}^{(1,1)}$ and $\mathbf{H}_{m}^{(2,1)}$ are diagonal matrices, and $\boldsymbol{X}_{m}$ and $\boldsymbol{X}_{m+1}$ are column vectors. By neglecting noise components in the above equation, estimates $\hat{\boldsymbol{X}}_{m}$ and $\hat{\boldsymbol{X}}_{m+1}$ of the transmitted information at receiver are

$$
\left[\begin{array}{c}
\hat{\boldsymbol{X}}_{m} \\
\hat{\boldsymbol{X}}_{m+1}
\end{array}\right]=\mathcal{H}_{m}\left[\begin{array}{c}
\boldsymbol{Y}_{m} \\
\boldsymbol{Y}_{m+1}^{*}
\end{array}\right]=\delta^{-1}\left[\begin{array}{cc}
\mathbf{H}_{m}^{(1,1) *} & \mathbf{H}_{m}^{(2,1)} \\
\mathbf{H}_{m}^{(2,1) *} & -\mathbf{H}_{m}^{(1,1)}
\end{array}\right]\left[\begin{array}{c}
\boldsymbol{Y}_{m} \\
\boldsymbol{Y}_{m+1}^{*}
\end{array}\right],
$$

where $\mathcal{H}_{m}$ is the Alamouti linear decoding matrix. This matrix is the inverse of the system matrix in (6), and its opposite determinant $\delta=\left(\left\|\mathbf{H}_{m}^{(1,1)}\right\|_{\mathcal{F}}^{2}+\left\|\mathbf{H}_{m}^{(2,1)}\right\|_{\mathcal{F}}^{2}\right)$ is the sum of the Frobenius norm of the respective channels. Explicitly, these estimates are, by inserting (6) in (7), given by

$$
\begin{gathered}
\hat{\boldsymbol{X}}_{m}=\delta^{-1}\left(\mathbf{H}_{m}^{(1,1) *} \boldsymbol{Y}_{m}+\mathbf{H}_{m}^{(2,1)} \boldsymbol{Y}_{m+1}^{*}\right)=\boldsymbol{X}_{m}+\boldsymbol{Z}_{m}^{\prime} \\
\hat{\boldsymbol{X}}_{m+1}=\delta^{-1}\left(\mathbf{H}_{m}^{(2,1) *} \boldsymbol{Y}_{m}-\mathbf{H}_{m}^{(1,1)} \boldsymbol{Y}_{m+1}^{*}\right)=\boldsymbol{X}_{m+1}+\boldsymbol{Z}_{m+1}^{\prime},
\end{gathered}
$$

where $Z_{m}^{\prime}$ and $Z_{m+1}^{\prime}$ are combination of noises filtered by the channel responses. Therefore, these estimates are the transmitted symbols plus noise components filtered by the communication links. The above combined signal-plus-noise components are then sent to the maximum likelihood detector.

\section{B. Transmitter Diversity in the Frequency Domain}

Diversity can also be exploited in the frequency domain [15]. Fig. 2 presents a block diagram of a system using orthogonal space-frequency block coding with two transmit and one receive antennas. This system is a simple extension of space-time coding to the frequency domain.

As in the case of transmitter diversity in the time domain, one OFDM engine is used for each transmitter antenna. After the serial-to-parallel conversion block, the data symbol $\boldsymbol{X}_{m}=$ $\left[X_{m}[0] \quad X_{m}[1] \quad \ldots \quad X_{m}[N-1]\right]^{T}$ is Alamouti coded into two matrices as follows

$$
\begin{gathered}
\mathbf{X}_{m}^{(1)}=\operatorname{diag}\left\{X_{m}[0],-X_{m}^{*}[1], \ldots, X_{m}[N-2], X_{m}^{*}[N-1]\right\}, \\
\mathbf{X}_{m}^{(2)}=\operatorname{diag}\left\{X_{m}[1], X_{m}^{*}[0], \ldots, X_{m}[N-1], X_{m}^{*}[N-2]\right\} .
\end{gathered}
$$

Unlike space-time diversity, in the same OFDM block instant $m, \mathbf{X}_{m}^{(1)}$ is transmitted from the first antenna while $\mathbf{X}_{m}^{(2)}$ is transmitted from the second antenna. For a better description of this modulation technique, it is useful to decompose the matrix $\mathbf{X}_{m}=\operatorname{diag}\left\{\boldsymbol{X}_{m}\right\}$ into its even and odd components using the following notation $\mathbf{X}_{m}^{e}, \mathbf{X}_{m}^{o}$. By doing the same for $\mathbf{X}_{m}^{(1)}$ and $\mathbf{X}_{m}^{(2)}$, we obtain $\mathbf{X}_{m}^{(1) e}, \mathbf{X}_{m}^{(1) o}, \mathbf{X}_{m}^{(2) e}$, and $\mathbf{X}_{m}^{(2) o}$. Thus, the equivalent space-frequency block coding matrix will be

$$
\mathbf{C}=\left[\begin{array}{cc}
\mathbf{X}_{m}^{e} & \mathbf{X}_{m}^{o} \\
-\mathbf{X}_{m}^{o *} & \mathbf{X}_{m}^{e *}
\end{array}\right]
$$

The received signal vector is $\boldsymbol{Y}_{m}=\mathbf{C} \boldsymbol{H}_{m}+\boldsymbol{Z}_{m}$. In a similar way to that in the previous section, we use a rearranged channel model

$$
\boldsymbol{Y}_{m}=\mathbf{H}_{m}^{(1,1)} \boldsymbol{X}_{m}^{(1)}+\mathbf{H}_{m}^{(2,1)} \boldsymbol{X}_{m}^{(2)}+\boldsymbol{Z}_{\boldsymbol{m}},
$$

where $\mathbf{H}_{m}^{(1,1)}$ and $\mathbf{H}_{m}^{(2,1)}$ are diagonal matrices whose diagonal elements are the respective unit responses of $h_{m}^{(1,1)}[n]$ and $h_{m}^{(2,1)}[n]$. Splitting $\boldsymbol{Y}_{m}$ into its even and odd components, and doing in similar way for matrices $\mathbf{H}_{m}^{(1,1)}$ and $\mathbf{H}_{m}^{(2,1)}$, we obtain

$$
\begin{gathered}
\boldsymbol{Y}_{m}^{e}=\mathbf{H}_{m}^{(1,1) e} \boldsymbol{X}_{m}^{(1) e}+\mathbf{H}_{m}^{(2,1) e} \boldsymbol{X}_{m}^{(2) e}+Z_{m}^{e} \\
\boldsymbol{Y}_{m}^{o}=\mathbf{H}_{m}^{(1,1) o} \boldsymbol{X}_{m}^{(1) o}+\mathbf{H}_{m}^{(2,1) o} \boldsymbol{X}_{m}^{(2) o}+Z_{m}^{o},
\end{gathered}
$$

where $Z_{m}^{e}$ and $Z_{m}^{o}$ are noise components. In similar way to space-time coding, using linear decoding Alamouti matrix and considering even and odd adjacent propagation channel responses as approximately equal, this conducts respectively to the estimates of $\hat{\boldsymbol{X}}_{m}^{e}$ and $\hat{\boldsymbol{X}}_{m}^{o}$ at the receiver:

$$
\begin{aligned}
& \hat{\boldsymbol{X}}_{m}^{e}=\delta^{-1}\left(\mathbf{H}_{m}^{(1,1) e *} \boldsymbol{Y}_{m}^{e}+\mathbf{H}_{m}^{(2,1) e} \boldsymbol{Y}_{m}^{o *}\right)=\boldsymbol{X}_{m}^{e}+\boldsymbol{Z}_{m}^{e^{\prime}} \\
& \hat{\boldsymbol{X}}_{m}^{o}=\delta^{-1}\left(\mathbf{H}_{m}^{(2,1) e *} \boldsymbol{Y}_{m}^{e}-\mathbf{H}_{m}^{(1,1) e} \boldsymbol{Y}_{m}^{o *}\right)=\boldsymbol{X}_{m}^{o}+\boldsymbol{Z}_{m}^{o^{\prime}},
\end{aligned}
$$

where $\delta=\left(\left\|\mathbf{H}_{m}^{(1,1) e}\right\|_{\mathcal{F}}^{2}+\left\|\mathbf{H}_{m}^{(2,1) e}\right\|_{\mathcal{F}}^{2}\right)$, and $Z_{m}^{e^{\prime}}$ and $Z_{m}^{o^{\prime}}$ are combinations of filtered noises by the channel responses. These terms are the even and odd components of the estimate $\hat{\boldsymbol{X}}_{m}$, which are sent to the maximum likelihood detector.

Other SF codes proposed in the literature guarantee full-rate and full-diversity transmission in MIMO-OFDM systems [18]. 


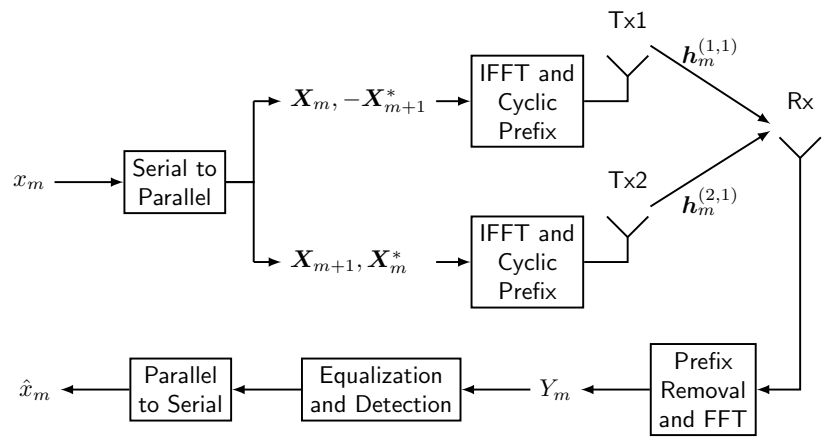

Fig. 1. Space-time OFDM transmit diversity system.

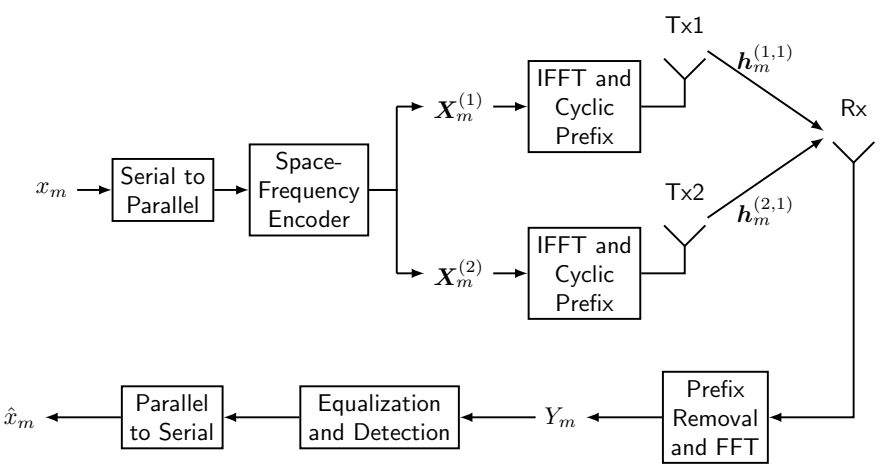

Fig. 2. Space-Frequency OFDM transmit diversity system.

\section{OFDM System USING QuATERnion Algebra}

The design of hypercomplex OFDM modulation schemes using quaternions and hypercomplex algebras were proposed by one of authors in [10]. These schemes use dual polarized antennas for complex signal transmission [8].

OFDM is based on the discrete Fourier transform. Several formulations of the Fourier transform have been defined for hypercomplex algebra. The first definition in context of nuclear magnetic resonance fields was proposed in the late 1980s [4]. For the quaternion OFDM scheme, results [10] show that the quaternion Fourier transform of 1D signals is suitable for this modulation technique

$$
X[k]=\sum_{n=0}^{N-1} e^{-\mu 2 \pi k n / N} x[n],
$$

where, in general, $\boldsymbol{\mu}$ is an arbitrary root of -1 , and $x[n]$ is a quaternion sequence. The quaternion OFDM transmitter system is illustrated in Fig. 3. In general, a forward error correction code can be applied to the binary sequence. Other important modules used in OFDM schemes, such as carrier and frame synchronizers, and time and frequency interleaving, can be included as need. At the transmitter input, a serial-toparallel conversion block stores $N$ samples for computing the inverse Fourier transform.

The quaternion $Q_{m}$ in the constellation uses the CayleyDickson (CD) form

$$
Q_{m}=Q_{m, 1}+Q_{m, 2} \boldsymbol{\mu},
$$

where $\boldsymbol{\mu}$ is either $\boldsymbol{i}, \boldsymbol{j}$, or $\boldsymbol{k}$, and $Q_{m, 1}$ and $Q_{m, 2}$ are complexes into orthogonal Argand domains to $\boldsymbol{\mu}$ and $Q_{m} \in \mathbb{H}$, respec- tively, (in the quaternion system). The simplex part of $Q_{m}$ is defined as $\mathcal{S}\left\{Q_{m}\right\}=Q_{m, 1}$ and the perplex part is defined as $\mathcal{P}\left\{Q_{m}\right\}=Q_{m, 2}$. The quaternion representation in the above form is called symplectic composition; it allows to resolve a quaternion into two perpendicular planes intercepting at the origin of the 4D space. Each of these planes is an Argand plane [5]. Incoming bit-streams are separated into two groups $b_{0, i}$ and $b_{1, i}$ (for $0 \leq i \leq N_{b}$ ); each of these groups defines an independent constellation, such that the entire constellation has $2^{2 N_{b}}$ entries [10]. It is possible to apply distinct modulations to different subcarriers.

The next block performs the inverse quaternion discrete Fourier transform, which can be implemented efficiently using classical fast Fourier transform algorithms [7], [19]. The complexity of these algorithms, without taking the complexity of the necessary Cayley-Dickson decomposition into account, is twice that of the classical Fourier transform.

The cyclic extended vector is prepared for transmission, which, in addition to providing subcarrier orthogonality, facilitates block synchronization. When using the time-shifting property of the quaternion Fourier transform, any consecutive $N$ samples of the OFDM symbol $m$ could be used; however, the last symbols, as indicated by (3), are better for mitigating inter-symbol interference (ISI).

The last blocks of the transmitter perform parallel-to-serial conversion, followed by the symplectic decomposition (conversion of quaternion into two complex numbers). In this way, the signals are transmitted by two independent antenna arrays using cross-polarized waves. The last transmitter blocks are typical in wireless communications systems; these blocks 


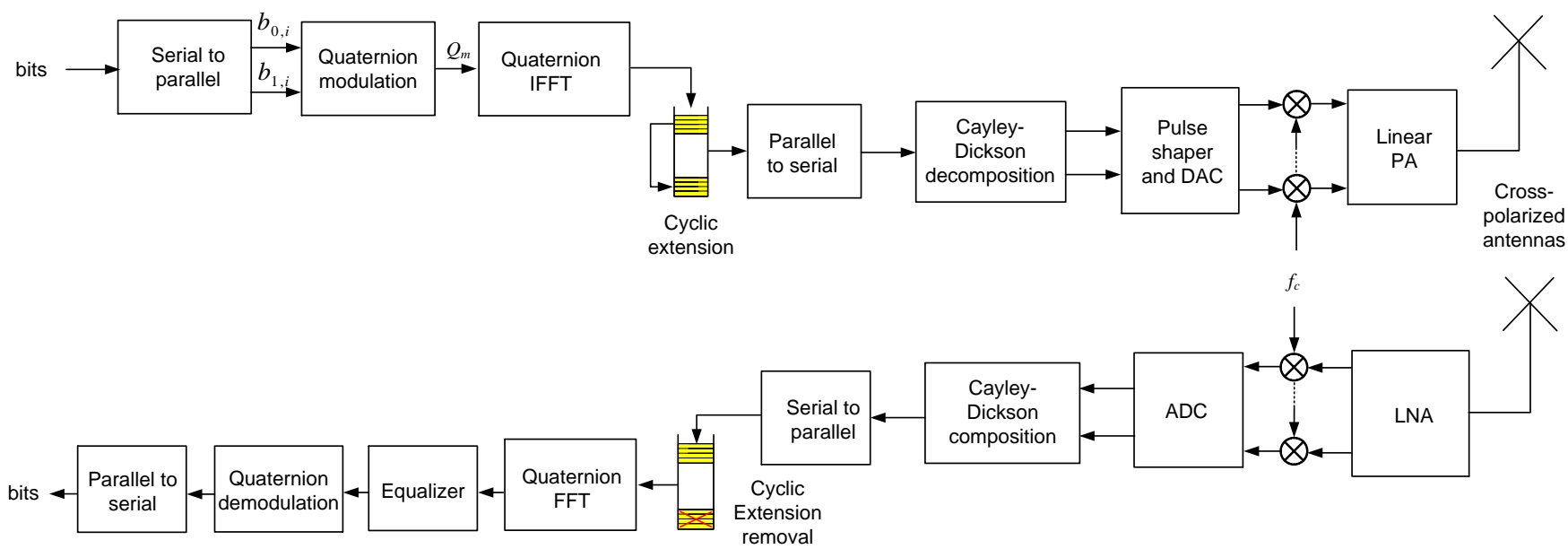

Fig. 3. Quaternion OFDM transmitter and receiver.

consist of a pulse shaper, a digital-to-analogue converter, a mixer, and power amplifier blocks.

At the receiver, after analogue-to-digital and down-conversion the first step is to compose the symplectic form using (9); next, data is buffered for performing the Fourier transform. First, cyclic extension is removed before applying the direct quaternion Fourier transform. The data equalization may be conducted in the Fourier domain, as illustrated in Fig. 3, but it might be applied in the time domain as well. The symplectic decomposition is performed inside the hypercomplex demodulation block; thereafter, a classical demodulator can be used.

\section{A. Cross-polarized Link Model}

It is important to mention that when using cross-polarization transmit and receive antennas, a link model of the channel will be [8], [9]

$$
H_{m}^{\times}=\left[\begin{array}{ll}
H_{m}^{\mathrm{hh}} & H_{m}^{\mathrm{hv}} \\
H_{m}^{\mathrm{vh}} & H_{m}^{\mathrm{vv}}
\end{array}\right],
$$

where each element has complex channel gains. $H_{m}^{\text {hh }}$ and $H_{m}^{\mathrm{vv}}$ are channel gains for the same polarization (for instance, horizontal or vertical), and $H_{m}^{\mathrm{hv}}$ and $H_{m}^{\mathrm{vh}}$ are gains for crosspolar scatter, received signals with different polarization due to reflection or twist between transmit and receive elements of the antennas. Using the following notation, which makes use of the Cayley-Dickson decomposition, a quaternion symbol $S_{m}=S_{m, 1}+S_{m, 2} \boldsymbol{\mu}$ can be represented as $\left[S_{m, 1}, S_{m, 2}\right]$, which are the simplex and perplex parts of $S_{m}$. After symbol transmission over the cross-polarized link defined by the above equation, the received quaternion symbol plus noise is $Y_{m}=S_{m} H_{m}^{\times}+Z$, or, explicitly,

$$
\begin{aligned}
Y_{m}= & {\left[Y_{m, 1}, Y_{m, 2}\right] } \\
= & {\left[S_{m, 1}, S_{m, 2}\right] H_{m}^{\times}+\left[Z_{1}, Z_{2}\right] } \\
= & {\left[\left(S_{m, 1} H_{m}^{\mathrm{hh}}+S_{m, 2} H_{m}^{\mathrm{vh}}\right),\left(S_{m, 1} H_{m}^{\mathrm{hv}}+S_{m, 2} H_{m}^{\mathrm{vv}}\right)\right] } \\
& +\left[Z_{1}, Z_{2}\right],
\end{aligned}
$$

where $Z_{1}$ and $Z_{2}$ are complex additive noise sources with identical variance.

\section{B. Link Characterisation of Dual-polarized Antenna}

Ideally, the cross-polar scatter elements $H_{m}^{\mathrm{hv}}$ and $H_{m}^{\mathrm{vh}}$ of (10) should be zero [17]; however, this does not hold true, because of two mechanisms of depolarization: the use of imperfect cross-polar isolation (XPI) antennas and the presence of wave scattering, represented by a cross-polar ratio (XPR) in the propagation channels. These effects are combined into an overall cross-polar discrimination parameter (XPD), which is defined as the ratio between the power in the orthogonal (or cross-polar) elements to the received power in the co-polar elements when the antenna is excited with a polarized wave in the co-polar element.

In simulations section, we will use a simple approximation to these mechanisms:

$$
H_{m}^{\times}=M_{r} H_{m}^{\perp} M_{t},
$$

where

$$
H_{m}^{\perp}=\left[\begin{array}{cc}
H_{m}^{\mathrm{hh}} & 0 \\
0 & H_{m}^{\mathrm{vv}}
\end{array}\right]
$$

represents a cross-polar channel with infinite XPI, and $M_{t}$ and $M_{r}$ represent coupling matrices at, respectively, transmit and receive sides by

$$
M_{t}=\gamma_{t}\left[\begin{array}{cc}
1 & \sqrt{\chi_{t}} \\
\sqrt{\chi_{t}} & 1
\end{array}\right] \text { and } M_{r}=\gamma_{r}\left[\begin{array}{cc}
1 & \sqrt{\chi_{r}} \\
\sqrt{\chi_{r}} & 1
\end{array}\right] \text {, }
$$

where scalar XPI is defined as $\chi_{t}^{-1}$ and $\chi_{r}^{-1}$ at transmit and receive antennas, respectively. We also assume that $H_{m}^{\text {hh }}$ and $H_{m}^{\mathrm{vv}}$ are independent channels. $\gamma_{t}$ and $\gamma_{r}$ are gain factors used for channel link normalisation, given by

$$
\gamma_{t}=\frac{\sqrt{2}}{1+\sqrt{\chi_{t}}} \quad \text { and } \quad \gamma_{r}=\frac{\sqrt{2}}{1+\sqrt{\chi_{r}}} .
$$

\section{QUATERNION MIMO-OFDM SYSTEM}

The proposed quaternion MIMO-OFDM (QOFDM) design uses an orthogonal space-time-polarization block (STP), space-frequency-polarization (SFP) block codes, or these two modes combined into space-time-frequency-polarization coding. Fig. 4 illustrates the quaternionic MIMO-OFDM system. 
At the transmitter, $M_{t}$ dual-polarized antennas are used. $M_{r}$ dual-polarized antennas are used at the receiver. In general, the frequency-selective fading channel for each pair TX-Rx has $L$ independent delay paths, and this channel remains constant over the QOFDM symbol period. The unit response from the transmit antenna $r$ to the receive antenna $s$ for each pair of elements of the cross-polarized antennas is

$$
h_{m}^{\bowtie(r, s)}[n]=\sum_{l=0}^{L} \alpha_{l}^{\bowtie(r, s)} \delta\left(n-n_{l}^{\bowtie}\right),
$$

where $\alpha_{l}^{\bowtie(r, s)}$ is a complex gain of the $l^{\text {th }}$ path between link $r$ and $s, n_{l}^{\bowtie}$ is the delay of the $l^{t h}$ path, and symbol $\bowtie$ is a mnemonic for $\mathrm{hh}, \mathrm{hv}$, vh, or vv polarization cases. The element-to-element channel frequency response is expressed by

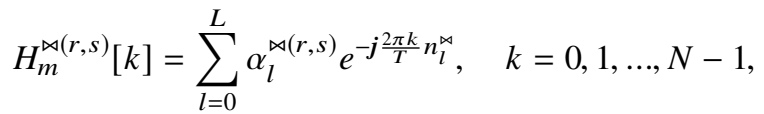

where the subcarrier separation is $1 / T$, and $j$ is the complex imaginary unit. In this way, a quaternion channel response from transmit antenna $r$ to receive antenna $s$ can be represented by quaternion vector

$$
\boldsymbol{H}_{m}^{\times(r, s)}=\left[H_{m}^{\times(r, s)}[0]^{\mathcal{T}} H_{m}^{\times(r, s)}[1]^{\mathcal{T}} \cdots H_{m}^{\times(r, s)}[N-1]^{\mathcal{T}}\right]^{\mathcal{T}} .
$$

Elements of $\boldsymbol{H}_{m}^{\times(r, s)}$ can also be separated into vectors for each link of the cross-polarized antennas

$$
\boldsymbol{H}_{m}^{\bowtie(r, s)}=\left[H_{m}^{\bowtie(r, s)}[0] H_{m}^{\bowtie(r, s)}[1] \cdots H_{m}^{\bowtie(r, s)}[N-1]\right]^{\mathcal{T}},
$$

which are link channel responses in (17).

The input bitstreams are pairwise separated into two groups $b_{0, i}$ and $b_{1, i}$, for $0 \leq i \leq N_{b}$, forming two $2^{N_{b}}$-ary complex symbols, which are mapped onto two perpendicular Argand planes intercepting at the origin of the 4D space [10]. Using the CD composition (9), quaternion values $Q_{m}[k]$, for $k=$ $0,1, \ldots, N-1$ are created. These $N$ quaternions are grouped into a column vector $\boldsymbol{Q}_{m}$ to be OFDM modulated using $N_{l}$ subcarriers.

A set of $M_{b}$ consecutive OFDM blocks, represented by $Q_{m}$, $\boldsymbol{Q}_{m+1}, \ldots, \boldsymbol{Q}_{m+M_{b}-1}$, are mapped onto a diversity codeword, which is expressed by the following $M_{b} N \times M_{t}$ matrix

$$
\mathbf{C}=\left[\begin{array}{llll}
C_{1}^{\mathcal{T}} & C_{2}^{\mathcal{T}} & \ldots & C_{M_{b}}^{\mathcal{T}}
\end{array}\right]^{\mathcal{T}},
$$

where each element $C_{m}$ is defined as

$$
C_{m}=\left[\begin{array}{cccc}
C_{m}^{(1)}[0] & C_{m}^{(2)}[0] & \ldots & C_{m}^{\left(M_{t}\right)}[0] \\
C_{m}^{(1)}[1] & C_{m}^{(2)}[1] & \ldots & C_{m}^{\left(M_{t}\right)}[1] \\
\vdots & \vdots & \ddots & \vdots \\
C_{m}^{(1)}[N-1] & C_{m}^{(2)}[N-1] & \ldots & C_{m}^{\left(M_{t}\right)}[N-1]
\end{array}\right] .
$$

The above $\boldsymbol{Q}_{m}$ mapping is similar to complex $\boldsymbol{X}_{m}$ mapping cases in Section II. Each column $l$ of matrix (20) will be transmitted from antenna $l$ after having been processed by an OFDM transmit engine, which applies a quaternionic IFFT (inverse fast Fourier transform) and appends a cyclic prefix. Frequency, time, or both frequency and time diversities are implemented along the rows of $\mathbf{C}$, whereas space and
TABLE I

DIMENSION OF SEVERAL QUATERNION MATRICES AND VECTORS FOR THE QUATERNIONIC MIMO-OFDM COMPUTED BASED ON THE NUMBER OF COMPLEX ELEMENTS.

\begin{tabular}{cc}
\hline Symbols & Dimensions \\
\hline $\mathbf{D}$ & $M_{b} N M_{r} \times 2 M_{b} N M_{t} M_{r}$ \\
$\mathcal{D}_{i}$ & $M_{b} N \times M_{b} N$ \\
$\boldsymbol{H}$ & $M_{b} N M_{t} M_{r} \times 2$ \\
$\boldsymbol{Y}, \boldsymbol{Z}$ & $M_{b} N M_{r} \times 2$ \\
\hline
\end{tabular}

polarization diversities are implemented along the columns of C. From the above matrix codeword, another sparse matrix is defined by

$$
\mathbf{D}=\mathbf{I}_{M_{r}} \otimes\left[\mathcal{D}_{1}^{s} \mathcal{D}_{1}^{p} \mathcal{D}_{2}^{s} \mathcal{D}_{2}^{p} \ldots \mathcal{D}_{M_{t}}^{s} \mathcal{D}_{M_{t}}^{p}\right],
$$

where $\otimes$ represents the Kronecker product, $\mathbf{I}_{M_{r}}$ is the identity matrix of order $M_{r}$, and elements $\mathcal{D}_{i}^{s}$ and $\mathcal{D}_{i}^{p}$ are diagonal matrices defined by

$$
\begin{aligned}
& \mathcal{D}_{i}^{s}=\operatorname{diag}\left\{\mathcal{S}\left\{C_{m}^{(i)}[0]\right\}, \mathcal{S}\left\{C_{m}^{(i)}[1]\right\}, \ldots,\right. \\
&\left.\mathcal{S}\left\{C_{m}^{(i)}\left[M_{b} N-1\right]\right\}\right\}, \quad i=1,2, \ldots, M_{t},
\end{aligned}
$$

and

$$
\begin{aligned}
& \mathcal{D}_{i}^{p}=\operatorname{diag}\left\{\mathcal{P}\left\{C_{m}^{(i)}[0]\right\}, \mathcal{P}\left\{C_{m}^{(i)}[1]\right\}, \ldots,\right. \\
&\left.\mathcal{P}\left\{C_{m}^{(i)}\left[M_{b} N-1\right]\right\}\right\}, \quad i=1,2, \ldots, M_{t},
\end{aligned}
$$

whose elements are columns of matrix C. Table I shows the dimension of the above vectors or matrices based on the number of complex elements.

The received signal in matrix form is

$$
\boldsymbol{Y}=\sqrt{\frac{\rho}{M_{t}}} \mathbf{D} \boldsymbol{H}+\boldsymbol{Z} .
$$

The $\sqrt{\rho / M_{t}}$ term is used for adjusting the signal-to-noise ratio at a receive antenna to be independent of the number of transmit antennas [18]. The channel $\boldsymbol{H}$ is defined as

$$
\begin{array}{r}
\boldsymbol{H}=\left[\boldsymbol{H}^{(1,1) \mathcal{T}} \cdots \boldsymbol{H}^{\left(M_{t}, 1\right) \mathcal{T}} \boldsymbol{H}^{(1,2) \mathcal{T}} \cdots \boldsymbol{H}^{\left(M_{t}, 2\right) \mathcal{T}}\right. \\
\left.\cdots \boldsymbol{H}^{\left(1, M_{r}\right) \mathcal{T}} \ldots \boldsymbol{H}^{\left(M_{t}, M_{r}\right) \mathcal{T}}\right]^{\mathcal{T}},
\end{array}
$$

where $\boldsymbol{H}^{(r, s)}$ is defined by

$$
\boldsymbol{H}^{(r, s)}=\left[\boldsymbol{H}_{m}^{\times(r, s) \mathcal{T}} \boldsymbol{H}_{m+1}^{\times(r, s) \mathcal{T}} \cdots \boldsymbol{H}_{m+M_{b}-1}^{\times(r, s)^{\mathcal{T}}}\right]^{\mathcal{T}},
$$

where $\boldsymbol{H}_{m}^{\times(r, s)}$ is defined in (18), and $m$ indicates the beginning of a block code.

The received signal is

$$
\boldsymbol{Y}=\left[\boldsymbol{y}^{(1) \mathcal{T}} \boldsymbol{y}^{(2) \mathcal{T}} \ldots \boldsymbol{y}^{\left(M_{r}\right) \mathcal{T}}\right]^{\mathcal{T}}
$$

where each element is

$$
\mathcal{Y}^{(i)}=\left[\begin{array}{c}
{\left[\mathcal{S}\left\{Y_{m}^{(i)}[0]\right\}, \mathcal{P}\left\{Y_{m}^{(i)}[0]\right\}\right]} \\
\vdots \\
{\left[\mathcal{S}\left\{Y_{m}^{(i)}[N-1]\right\}, \mathcal{P}\left\{Y_{m}^{(i)}[N-1]\right\}\right]} \\
{\left[\mathcal{S}\left\{Y_{m+1}^{(i)}[0]\right\}, \mathcal{P}\left\{Y_{m+1}^{(i)}[0]\right\}\right]} \\
\vdots \\
{\left[\mathcal{S}\left\{Y_{m+M-1}^{(i)}[N-1]\right\}, \mathcal{P}\left\{Y_{m+M-1}^{(i)}[N-1]\right\}\right]}
\end{array}\right] .
$$




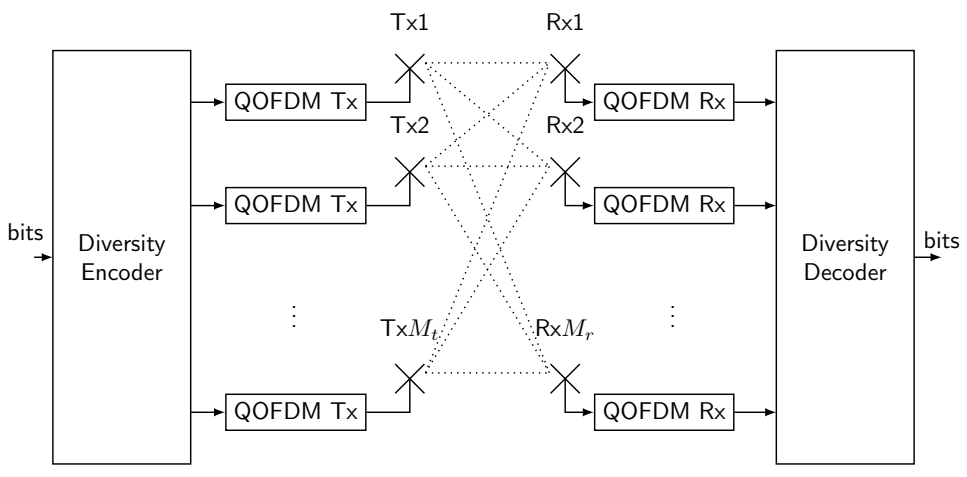

Fig. 4. Quaternion MIMO-OFDM system using cross-polarized antennas.

The structure of the quaternion noise vector $Z$ is similar to that given above for $\boldsymbol{Y}$. The simplex and perplex parts of the quaternion noise $\boldsymbol{Z}$ are complex additive Gaussian noise with independent and identically distributed zero-mean 2D Gaussian random variables with the same variance. For the presented formulation, once one of the cross-polarized elements are turned off, the equations are reduced to classical complex schemes [20].

\section{A. Orthogonal Diversity Codes}

The proposed MIMO-OFDM technique presented in the previous section can take advantage of the diversity in several dimensions, i.e. space, time, frequency, and polarization. The first space-time code in OFDM modulation made use of trellis codes [21]. Unlike space-time block codes (STBCs), trellis codes are able to provide both coding gain and diversity gain, and have better bit-error rate performance; however, they are more complex than STBCs, because they rely on a Viterbi decoder at the receiver, whereas STBC uses only linear processing. Studies have shown that STBC, which achieves full diversity in quasi-static flat fading channels, can be used to construct space-frequency codes that achieve the maximum diversity available in frequency selective MIMO fading channels [20].

A theory for quaternion orthogonal designs was developed in [9]. Several details related to this work are reproduced here, where it is relevant to know whether the quaternion orthogonal designs are built over complex or quaternion variables. Quaternion orthogonal design (QOD) may be defined over complex variables commutative for multiplication $z_{1}, \ldots, z_{u}$ of type $\left(s_{1}, \ldots, s_{u}\right)$ as an $r \times n$ matrix $\mathbf{A}$ with entries from a set $\left\{0, \pm z_{1}, \pm z_{1}^{*}, \pm z_{2}, \pm z_{2}^{*}, \ldots, \pm z_{u}, \pm z_{u}^{*}\right\}$, including possible multiplications on the left and/or right by a quaternion element $q \in \mathbb{H}$, and satisfying

$$
\mathbf{A}^{\mathcal{H}} \mathbf{A}=\left(\sum_{l=1}^{u} s_{l}\left|z_{l}\right|^{2}\right) \mathbf{I}_{n},
$$

where $(\cdot)^{\mathcal{H}}$ indicates the Hermitian quaternion matrix transpose and $\mathbf{I}_{n}$ is the identity matrix of order $n$.

Similarly, we define a QOD A on non-commutative quaternion variables $a_{1}, a_{2}, \ldots, a_{u}$ to have entries from a set $\left\{0, \pm a_{1}, \pm a_{1}^{*}, \pm a_{2}, \pm a_{2}^{*}, \ldots, \pm a_{u}, \pm a_{u}^{*}\right\}$, including possible multiplications on the left and/or right by quaternion element $q \in \mathbb{H}$, and satisfying $\mathbf{A}^{\mathcal{H}} \mathbf{A}=\left(\sum_{l=1}^{u} s_{l}\left|a_{l}\right|^{2}\right) \mathbf{I}_{n}$.

The fact that $\mathbf{A}^{\mathcal{H}} \mathbf{A}$ is diagonal ensures decorrelation of the signals transmitted at the receiver. An orthogonal design that has entries that are linear combinations of the permitted variables of the respective sets is said to be a design that performs linear processing. Calderbank et al. proposed a quaternion based QOD [22], but it was not with linear processing.

The QOD suggested in [9] takes two CODs $(2,2)$ (complex orthogonal design) that are equivalent designs to Alamouti's code [23]

$$
\mathbf{A}=\left[\begin{array}{cc}
z_{1} & z_{2} \\
-z_{2}^{*} & z_{1}^{*}
\end{array}\right] \quad \text { and } \quad \mathbf{B}=\left[\begin{array}{cc}
z_{2} & z_{1} \\
z_{1}^{*} & -z_{2}^{*}
\end{array}\right]
$$

such that applying the Cayley-Dickson construction over the matrices results in

$$
\mathbf{C}=\mathbf{A}+\mathbf{B} \boldsymbol{j}=\left[\begin{array}{cc}
z_{1}+z_{2} \boldsymbol{j} & z_{2}+z_{1} \boldsymbol{j} \\
-z_{2}^{*}+z_{1}^{*} \boldsymbol{j} & z_{1}^{*}-z_{2}^{*} \boldsymbol{j}
\end{array}\right]
$$

which is a $\operatorname{QOD}(2,2)$ with linear processing on complex variables $z_{1}$ and $z_{2}$.

In this study, to take advantage of several diversities, it will be of interest to work with orthogonal designs of higher order. One example of QOD $(4,4)$ with linear processing on complex variables $z_{1}, z_{2}$, and $z_{3}$ that uses Cayley-Dickson construction is

$$
\mathbf{C}=\left[\begin{array}{cccc}
z_{3}-z_{1} \boldsymbol{j} & -2 z_{2} \boldsymbol{j} & -z_{1}+z_{3} \boldsymbol{j} & -2 z_{2} \\
2 z_{2}^{*} \boldsymbol{j} & z_{3}-z_{1}^{*} \boldsymbol{j} & 2 z_{2}^{*} & -z_{1}^{*}+z_{3} \boldsymbol{j} \\
z_{1}^{*}+z_{3}^{*} \boldsymbol{j} & -2 z_{2} & z_{3}^{*}+z_{1}^{*} \boldsymbol{j} & -2 z_{2} \boldsymbol{j} \\
2 z_{2}^{*} & z_{1}+z_{3}^{*} \boldsymbol{j} & 2 z_{2}^{*} \boldsymbol{j} & z_{3}^{*}+z_{1} \boldsymbol{j}
\end{array}\right]
$$

Simplex and perplex parts of quaternion vectors $\boldsymbol{Q}_{m}, \boldsymbol{Q}_{m+1}, \ldots, \boldsymbol{Q}_{m+M_{b}-1}$ define complexes $z_{i}$ in codeword $\mathbf{C}$.

In [9], the QODs obtained over quaternion variables are found to be more general and better suited to achieving full diversity gain for an orthogonal and full rate code. In addition to the CD construction, other methods for designing QODs are quaternion-commuting variables and quaternion amicable designs. However, for the purposes of this paper, because of its simplicity, the CD construction technique was applied. 


\section{B. Maximum Likelihood Decoding}

For most part of simualations in the next section, maximum likelihood (ML) detection is used. For a given channel (known at the receiver), ML detector is based on the minimum Euclidean distance between the received signal and all possible transmit symbols. The channel estimation at the receiver is typically implemented using channel equalizers from special symbols (long prefix) that are periodically transmitted or from the pilot subcarriers. Thus, the ML detector aims to minimise the norm of $\|\boldsymbol{Y}-\mathbf{C H}\|$. This process is equivalent to finding a set of symbols $\hat{\mathbf{C}}$ that minimises the squared norm

$$
\begin{aligned}
\|\boldsymbol{Y}-\mathbf{C} \boldsymbol{H}\|^{2}= & \operatorname{tr}\left\{(\boldsymbol{Y}-\mathbf{C} \boldsymbol{H})^{\mathcal{H}}(\boldsymbol{Y}-\mathbf{C} \boldsymbol{H})\right\} \\
= & \operatorname{tr}\left\{\left(\boldsymbol{Y}^{\mathcal{H}}-\boldsymbol{H}^{\mathcal{H}} \mathbf{C}^{\mathcal{H}}\right)(\boldsymbol{Y}-\mathbf{C} \boldsymbol{H})\right\} \\
= & \operatorname{tr}\left\{\boldsymbol{Y}^{\mathcal{H}} \boldsymbol{Y}-\boldsymbol{Y}^{\mathcal{H}} \mathbf{C} \boldsymbol{H}-\right. \\
& \left.\boldsymbol{H}^{\mathcal{H}} \mathbf{C}^{\mathcal{H}} \boldsymbol{Y}+\boldsymbol{H}^{\mathcal{H}} \mathbf{C}^{\mathcal{H}} \mathbf{C} \boldsymbol{H}\right\} \\
= & \operatorname{tr}\left\{\boldsymbol{Y}^{\mathcal{H}} \boldsymbol{Y}\right\}-2 \operatorname{Re}\left\{\operatorname{tr}\left[\boldsymbol{Y}^{\mathcal{H}} \mathbf{C} \boldsymbol{H}\right]\right\}+ \\
& \lambda \operatorname{tr}\left\{\boldsymbol{H}^{\mathcal{H}} \boldsymbol{H}\right\},
\end{aligned}
$$

where in the last term $\mathbf{C}^{\mathcal{H}} \mathbf{C}=\lambda \mathbf{I}$. For a circular constellation, $\boldsymbol{Y}^{\mathcal{H}} \boldsymbol{Y}$ is independent of the transmitted codeword.

\section{Simulation Results}

Performance analysis of the quaternion MIMO-OFDM systems was carried out considering several common channel scenarios: flat channel and selective fading (time and frequency selectivity). In the strict sense, time and frequency spreading in a mobile channel are correlated, but it is common practice to independently analyse these effects, following the WSSUS (wide-sense stationary uncorrelated scattering) assumption [24].

This section present simulations for complex and quaternion OFDM, where for all them $N_{l}=100$ and quadrature phaseshift keying (QPSK) is used per subcarrier modulation. Experiments were conducted using $\operatorname{QOD}(2,2)$ in $(27)$ and $\operatorname{QOD}(4,4)$ in (28). The power normalization for the transceiver antennas is the same as those used by Alamouti [23], where there are four radiating elements (two cross-polarized elements) for the $\operatorname{QOD}(2,2)$ and eight elements for $\operatorname{QOD}(4,4)$ are considered. The presented results are based on average bit error rate (BER) curves as a function of the signal-to-noise ratio (SNR) per bit.

In the first simulation scenario, channel coefficients were assumed to be known at the receiver and are constant in each OFDM block containing $M_{b}$ symbols. Channel coefficients are generated as random complex Gaussian i.d.d. variables, with zero mean and unit variance. The results shown in Fig. 5 indicate that for this flat fading Rayleigh channel with AWGN, using QPSK modulation, quaternion orthogonal space-timepolarization block coding (QOSTPBC) transmit systems, and for instance at a BER line of $10^{-3}$, substantial diversity gains relative to established techniques are obtained, such as confronting the classic Alamouti STBC scheme. SNR gains are found to be over $7 \mathrm{~dB}$ for $\operatorname{QOD}(2,2)$, and over $10 \mathrm{~dB}$ for $\mathrm{QOD}(4,4)$, these gains are due to the exploration of polarization diversity. We observe that the results presented

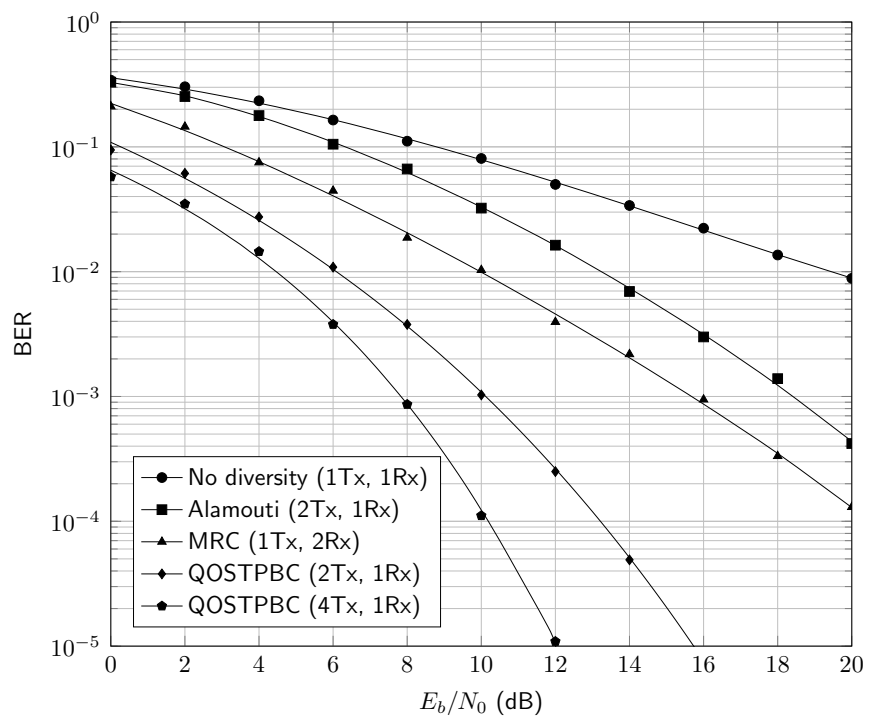

Fig. 5. Diversity gain for space-time-polarization transmit systems over a flat fading Rayleigh channel: first three upper curves consider single elements for $\mathrm{Tx}$ and Rx complex cases, and dual polarization antennas (two elements) for the QOSTBC cases.

for $\mathrm{QOD}(2,2)$, although using MIMO-OFDM, are very similar to those presented in [9].

In the second simulations in Fig. 6, we considered quaternion orthogonal space-time-frequency-polarization block coding (QOSTFPBC) using QOD $(4,4)$ for both mobile and static receivers (i.e. accounting for the Doppler shift or not). Regarding diversity, pairs of columns of $\mathbf{C}$ in (28) are grouped for space-frequency-polarization diversity, and pairs of rows of $\mathbf{C}$ are grouped for space-time-polarization diversity. Simulations consider that the unit responses $h_{m}^{\bowtie(r, s)}[n]$ in (16) are independent for hh, hv, vh, and vv links. A maximum Doppler shift of $f_{d}=100 \mathrm{~Hz}$ was chosen for representation of relative automobile speeds in the urban environment in the GSM band. For a total bandwidth of $1 \mathrm{MHz}$, the following four Rayleigh channels were simulated [25]: COST 207, typical urban case with a six-ray profile (TUx6), with and without Doppler shift; and COST 207, typical urban case with twelve-ray profile (TUx12), with and without mobile unit speed. When Doppler shift was considered, it was modelled as a classic Jakes' Doppler spectrum. Channel models in Matlab standard fading functions are used to compute the frame-to-frame unit response for each OFDM symbol. This process yields $\boldsymbol{H}$, which is to be applied to symbol codewords according to (22). Although transmission channels are, in theory, assumed to be static over the entire codeword $\mathbf{C}$, vector $\boldsymbol{Y}$ will contain the channel evolution over $M_{b}$ OFDM symbols and will be degraded by noise components. By contrast, in ML decoding, $\boldsymbol{H}$ is assumed to be static and equal to $\boldsymbol{H}_{m}$, corresponding to the first OFDM symbol $m$. This situation simulates the channel equalizer in periods equal to the codeword duration and is updated only at the next codeword. Results for QOSTFPBC transmit systems show good robustness for these different wireless communication scenarios. We observe that, for a BER line of $10^{-5}$, there is a difference between the typical urban 


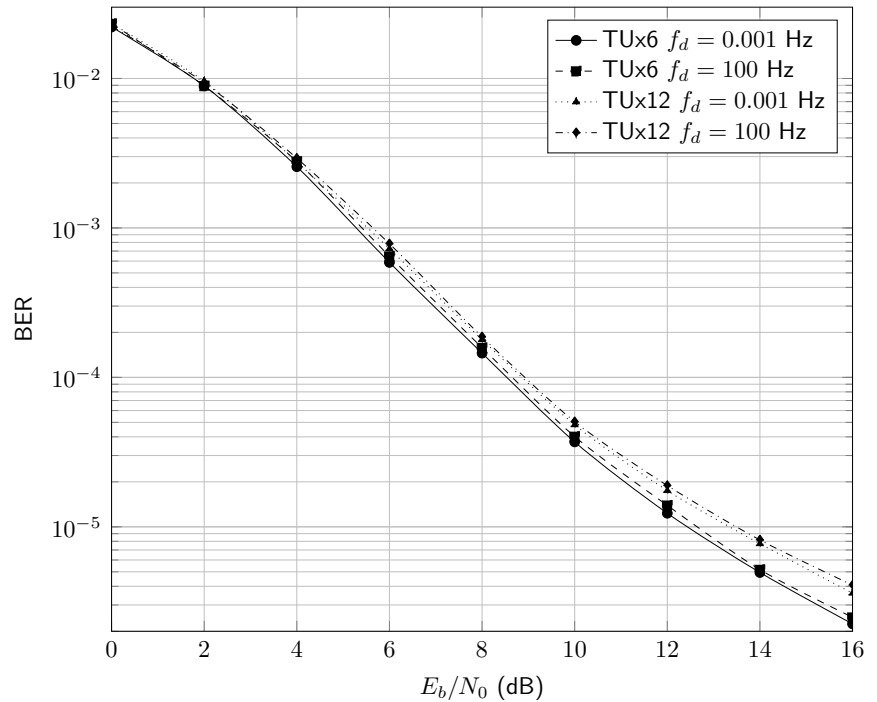

Fig. 6. BER for $\mathrm{Q}(4,4)$ QOSTFPBC for different wireless communications scenarios.

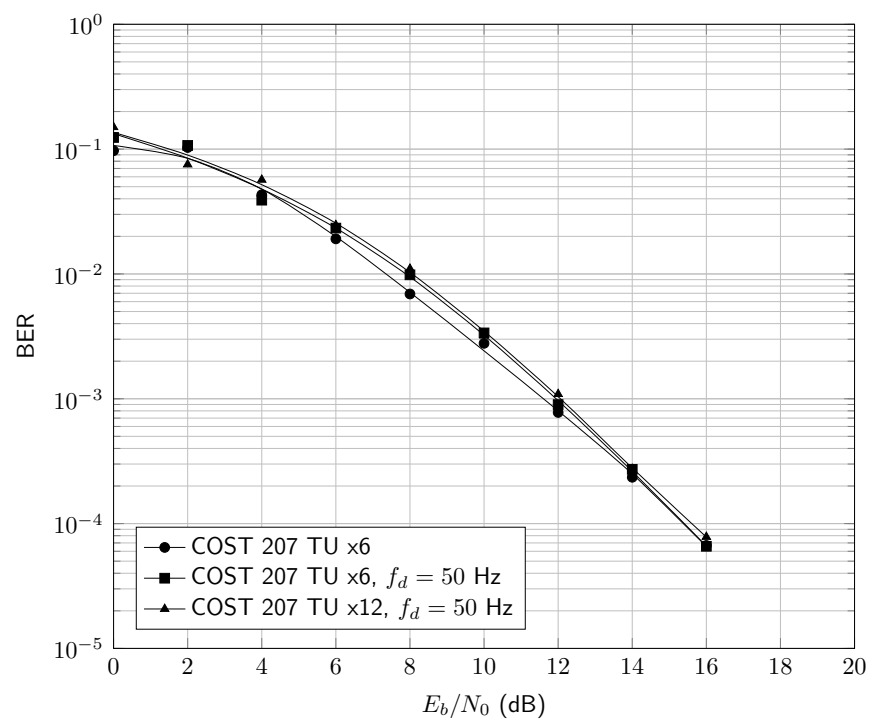

Fig. 7. BER for $\mathrm{Q}(4,4)$ QOSTFPBC using scattering matrix in different wireless communications scenarios.

case with a six-ray profile using $f_{D}=0.001 \mathrm{~Hz}$ and the twelveray profile using $f_{D}=100 \mathrm{~Hz}$ is $1.1 \mathrm{~dB}$. Good discrimination among the four propagation scenarios is observed. When compared with the same QOD $(4,4)$ scheme of Fig.5, moderate diversity gains (around $3 \mathrm{~dB}$ ) are observed for $E_{b} / N_{0}$ up to 8 $\mathrm{dB}$, even not considering the channel state information at the transmitter side (CSIT) or more sofisticated schemes, such as those using beamforming antenna arrays [26].

The final simulations in Fig. 7 are similar to those of the previous scenario; however, they use matrix (12) for different values of parameter XPI at receive and transmit antennas: $\chi_{t}=\chi_{r}=0$ and $\chi_{r}$, and $\chi_{t}=\chi_{r}=0.01$, without provision of any mechanism of cross-polarization interference cancelation (XPIC). As long as this cross paths are unknown in the receiver, a degradation in the performance is observed around $6 \mathrm{~dB}$ for same considered BER.
Simulations show that QOSTFPBC can effiently be used for flexible higher spectral efficency designs, as long as the channel state information is kwown at receivers (CSIR). As observed in [9], when using well-designed quaternion orthogonal codes, and here by use of quaternion MIMOOFDM, quaternion schemes are capable of achieving the same performance as complex orthogonal space-time-block codes or complex orthogonal space-frequency-block codes, which would require twice the number of transmit and receive antennas.

The last two simulations show that it is possible to consider the communications schemes using independent antenna elements with spatial separation, which represents a doubling in the number of $M_{t}$ and $M_{r}$ antennas at the transmit and receive sides, or using polarized antennas. Therefore, it is possible to group pairs of antennas on both sides to apply the proposed formulation with non-polarized antennas. By contrast, dualpolarized antennas have the advantage of reducing the size of transmit and receive antennas.

\section{CONCLUSIONS}

This paper has introduced a formulation for MIMO-OFDM using quaternion algebra. The formulation is general and allows us to take advantage of diversities in several domains, such as space, time, frequency, and polarization. The use of orthogonal quaternion code designs of higher order allows us to explore full diversity in those domains. Several simulations exploring scenarios in typical and severe urban environments, including the Doppler shift in the Jakes spectral model, have been presented. As expected, the use of a higher number of transmit antennas significantly improves the system performance, as could be observed for scenarios using random coefficients for the link frequency responses. Using models for real-world simulations (COST207), good discrimination of the propagation scenarios can be observed in simulations that are coherent to channel harshness.

The presented formulation applies both to double-polarized antennas and to independent antenna elements that are spatially separated. The presented modulation systems have potential applications in modern wireless communications including next-generation mobile, back-hauling, and digital television systems.

\section{ACKNOWLEDGEMENT}

The authors gratefully acknowledge the financial support of Capes, the research funding agency of the Brazilian government.

\section{REFERENCES}

[1] W. Wood, How Apollo Flew to the Moon. New York, NY: Springer, 2011, doi: 10.1007/978-1-4419-7179-1.

[2] B. Friedland, "Analysis of strapdown navigation using quaternions," IEEE Trans. Aerospace Electronic Systems, vol. 43(3), pp. 764-768, September 1978, doi: 10.1109/TAES.1978.308627.

[3] T. Ell, "Hypercomplex spectral transformations," Ph.D. dissertation, University of Minnesota, 1992. [Online]. Available: http://search.proquest.com/docview/304027651/abstract

[4] M. A. Delsuc, "Spectral representation of 2D NMR spectra by hypercomplex numbers," Journal of Magnetic Resonance, vol. 77(1), pp. 119124, March 1988, doi: 10.1016/0022-2364(88)90036-4. 
[5] S. Sangwine and T. Ell, "The discrete Fourier transform of a colour image," Second IMA Conf. on Image Process., pp. 430-441, September 1998.

[6] N. Bihan, S. Sangwine, and T. Ell, "Instantaneous frequency and amplitude of orthocomplex modulated signals based on quaternion Fourier transform," Signal Process., vol. 94, pp. 308-318, 2014, doi: 10.1016/j.sigpro.2013.06.028.

[7] T. Ell and S. Sangwine, "Hypercomplex Fourier transform of colour images," IEEE Trans. Image Process., vol. 16(1), pp. 22-35, January 2007, doi: 10.1109/ICIP.2001.958972.

[8] B. J. Wysocki, T. A. Wysocki, and J. Seberry, "Modeling dual poralization wireless fading channels using quaternions," Proc. Symp. on Trends in Communications, vol. 1, no. 1, pp. 68-71, 2006, doi: 10.1109/TIC.2006.1708024.

[9] J. Seberry, K. Finlayson, S. S. Adams, T. A. Wysocki, T. Xia, and B. J. Wysocki, "The theory of quaternion orthogonal designs," IEEE Trans. on Signal Processing, vol. 56, pp. 256-265, 2008, doi: 10.1109/TSP.2007.906773.

[10] L. Meloni, "Hypercomplex OFDM schemes for cross-polarized antennas," 2012 International Symposium on Communications and Information Technologies, vol. 1, no. 1, pp. 1-6, 2012, doi: 10.1109/ISCIT.2012.6380951.

[11] G. Raleigh and V. Jones, "Multivariate modulation and coding for wireless communication," IEEE Journal on Selected Areas in Communications, vol. 17, no. 5, pp. 851-866, May 1999.

[12] "IEEE Standard for Information technology Local and metropolitan area networks Specific requirements Part 11: Wireless LAN Medium Access Control (MAC)and Physical Layer (PHY) Specifications Amendment 5: Enhancements for Higher Throughput," pp. 1-565, 2009.

[13] Y. S. Cho, J. Kim, W. Y. Yang, and C. G. Kang, MIMO-OFDM Wireless Communications with MATLABß. Chichester, UK: John Wiley \& Sons, Ltd, aug 2010.

[14] K. Lee and D. Williams, "A space-time coded transmitter divesity technique for frequency selective fading channels," Proc. IEEE Sensor Array and Multichannel Signal Processing Workshop, Cambridge,MA, vol. 1, no. 1, pp. 149-152, 2000, doi: 10.1109/SAM.2000.877987.

[15] _ _A space-Frequency Transmitter Diversity Technique for OFDM Systems," Proc. Global Telecommun. Conference, Globecom, San Francisco, $C A$, vol. 3 , no. 1, pp. 1474-1477, 2000, doi: 10.1109/GLOCOM.2000.891885.

[16] K. Murayama, "Large-capacity transmission technology for nextgeneration digital television broadcasting in Super Hi-Vision," $N H K$ STRL $R \& D$, vol. 134, pp. 26-34, 2012.

[17] C. Oestges, B. Clerckx, M. Guillaud, and M. Debbah, "Dual-polarized communications: From propagation models to syspem performance evaluation," IEEE Trans. on Wireless Communications, vol. 7, pp. 40194031, 2008, doi: 10.1109/T-WC.2008.070540.

[18] W. Su, Z. Safar, and K. J. R. Liu, "Full-Rate Full Diversity SpaceFrequency Codes with Optimum Coding Advantage," IEEE Trans. on Information Theory, vol. 51, no. 1, pp. 229-249, 2005, doi: 10.1109/TIT.2004.839496.

[19] S. Said, N. Le Bihan, and S. Sangwine, "Fast complexified quaternion fourier transform," IEEE Trans. on Signal Processing, vol. 56, no. 4 pp. 1522-1531, 2008, doi: 10.1109/TSP.2007.910477.

[20] W. Su, Z. Safar, M. Olfat, and K. J. R. Liu, "Obtaining Full-Diversity Space-Frequency Codes From Space-Time Codes via Mapping," IEEE Trans. on Signal Process., vol. 51, no. 11, pp. 2905-16, 2003, doi: 10.1109/TSP.2003.818200.

[21] D. Agrawal, V. Tarokh, A. Naguib, and N. Seshadri, "Space-time coded ofdm for high data-rate wireless communication over wideband channels," Proc. IEEE Vehic. Tech. Conf., pp. 2232-36.

[22] A. R. Calderbank, S. Diggavi, S. Das, and N. Al-Dhahir, "Construction and analysis of a new 4 x 4 orthogonal space-time block code," in Information Theory, 2004. ISIT 2004. Proceedings. International Symposium on, June 2004, doi: 10.1109/ISIT.2004.1365346, pp. 309309.

[23] S. Alamouti, "A simple transmit diversity technique for wireless communications," IEEE J. Sel. Areas Commun., vol. 16, no. 8, pp. 1451-1458, 1998, doi: 10.1109/49.730453.

[24] P. Bello, "Characterization of Randomly Time-Variant Linear Channels," IEEE Trans. Commun., vol. 11, no. 4, pp. 360-393, dec 1963, doi: 10.1109/TCOM.1963.1088793.

[25] M. Failli and COST 207 Management Committee, COST 207: Digital Land Mobile Radio Communications. European Comission, 1989.

[26] G. Caire, N. Jindal, M. Kobayashi, and N. Ravindran, "Multiuser MIMO Achievable Rates With Downlink Training and Channel State Feedback,"
IEEE Transactions on Information Theory, vol. 56, no. 6, pp. 28452866, Jun 2010

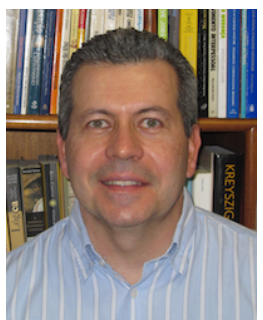

Luís Geraldo Pedroso Meloni is professor at the School of Electrical and Computer Engineering (FEEC) at University of Campinas (UNICAMP), Brazil, Ph.D. at the Universit de Nancy I (1985), France, Master of Science (1982) and undergraduation (1980) in Electrical Engineering, both at UNICAMP. He has a large academic and industrial experience in telecommunications. He has been member of the Council of the Brazilian System for Digital Television Forum since 2012, coordinating works of standardization of technologies for interactive channel. He has been the Coordinator of the Innovation and Technology Sector at FEEC for the past four years. He has been working in the Digital Signal Processing since 1982, and before Academia has worked previously in several telecommunications companies. He was lecturer at University of Brasilia (1990-1993) and currently at the University of Campinas. He has been working at the Real Time Digital Signal Processing Laboratory in several research areas: new technologies for wireless communications, softwaredefined radio, digital television technologies for interactive channel, speech and audio coding, voice over IP, speech recognition, development of software tools for education and learning via Internet. He has many publications in international journals and symposia and also has been instructor in continuing Education programs for telecommunications professionals.

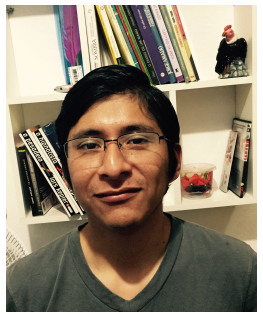

José Luis Hinostroza N. received the B.Sc. in Telecommunications Engineering from Ponthifical Catholic University of Perú (PUCP), Perú, in 2008, the M.Sc. degree in Electrical Engineering from University of Campinas (UNICAMP), Brazil, in 2015. He is currently pursuing the Ph.D. degree in Electrical Engineering also at UNICAMP. His research interests are communication theory, wireless communicatications, and application of hipercomplex algebras in communications systems.

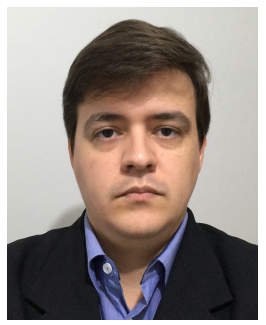

Osmar Tormena Junior received a B.Sc. and a M.Sc. degree in Electrical Engineering from the State University of Londrina (UEL), Brazil, in 2008 and 2010, respectively. Currently, he is pursuing his Ph.D., also in Electrical Engineering, from the University of Campinas (UNICAMP), Brazil. In 2012, he became assistant professor at Federal Technological University of Paraná (UTFPR). His research interests include application of hypercomplex algebras-particularly quaternions-in signal processing and telecomunications. 